\title{
Supercover of Straight Lines, Planes and Triangles
}

\author{
Eric Andres $(*)$, Philippe Nehlig (**), and Jean Françon (**) \\ (*) TUCS - Turku Centre for Computer Science \\ Lemminkäisenkatu 14A, 20520 Turku, Finland \\ $\left.{ }^{(* *}\right)$ Laboratoire des Sciences de l'Image, de l'Informatique et de la Télédétection \\ Université Louis Pasteur \\ 7 rue René Descartes, 67084 Strasbourg Cedex, France \\ (andres, nehlig, francon\}edpt-info.u-strasbg.fr
}

\begin{abstract}
The Supercover of a Euclidean object is the set of the pixels or voxels intersected by the object. The Supercover of $2 \mathrm{D}$ lines and $2 \mathrm{D}$ triangles are defined analytically. Some geometric properties, localization, and generation algorithms are given. The same is done for 3D lines, planes, and 3D triangles.
\end{abstract}

Keywords: Discrete 3D Modelling, Discrete Lines, Discrete Planes, Discrete Polygons, Supercover.

\section{Introduction}

The Supercover of a continuous (Euclidean) object is the set of the pixels or voxels intersected by the object, where a pixel (resp. voxel) is a unit square (resp. cube) of $\mathbf{R}^{2}$ (resp. $\mathbf{R}^{3}$ ) centered on a point of integer coordinates (integer point) of $\mathbf{Z}^{2}$ (resp. $\mathbf{Z}^{3}$ ), the discrete plane (resp. space). This concept of discretization is not new. Recently, Cohen and Kaufman [Cohe95] reactivated this concept for volume graphics purposes. In fact, supercovers have interesting properties of tunnel-freeness, and interesting properties under set operations. More recently, [ASA96] introduces for the first time discrete analytical descriptions of the Supercover of a $2 \mathrm{D}$ line defined by two rational points, and of a $3 D$ plane defined by three non-colinear rational points. Thus, discrete analytical modelling becomes possible.

While in [ANF97] we extended [ASA96] by introducing discrete analytical descriptions of the Supercover of a 3D line, of a polygon, and of a polyhedron for discrete geometric modelling purposes, giving their generation algorithms, in the present paper, we develop systematically the geometric properties of the Supercover of a $2 \mathrm{D}$ line, of a $2 \mathrm{D}$ triangle, of a 3D line, of a 3D plane, and of a 3D triangle. We develop also the basic generation algorithms as well as point localizing algorithms. The complexity of our algorithms, in the general case, is proportional to the number of generated points, and can be improved using usual optimisation techniques.

The paper is organised as follows. In section 2 we present the notations and the main definitions used in this paper. In section 3.1 we give formula for the Supercover of points and of boxes; in section 3.i, for $i=2,3$. we deal with the definition, properties and algorithms of the supercovers of primitives of dimension i. We conclude in section 4 . 
The algorithms are elementary consequences of the main theorems. The main theorems are the characterization of the Supercover of :

(1) a 2D straight line (resp. 3D plane) by an arithmetic line (resp. plane);

(2) a 3D straight line by the Supercover of its 3 projections on the orthotropic planes:

(3) a $2 \mathrm{D}$ or a $3 \mathrm{D}$ triangle by set operations on the preceding supercovers.

The proofs are either very elementary or long, technical, and without giving more light on the asserted propositions. We give thus only sketches for some of them.

\section{Preliminaries}

The set of the real numbers is noted $\mathbf{R}$, the set of the rational numbers $\mathbf{Q}$, the set of the integers $Z$, and the set of the strictly positive integers $N^{*} .\lfloor x\rfloor$ is the greatest integer less than or equal to $x .\lceil x\rceil$ is the least integer greater than or equal to $x$. If not specified differently, $\left\{\frac{p}{q}\right\}$, where $\frac{p}{q}$ is rational, is a Euclidean remainder $\left\{\frac{p}{q}\right\}=p-q\left\lfloor\frac{p}{q}\right\rfloor$. The greatest common divisor of the integers $a_{1}, \ldots, a_{n}$ is noted $\operatorname{gcd}\left(a_{1}, \ldots, a_{n}\right)$. The dimension of the space is noted $n$. In this article we limit ourselves to dimension 1 to 3 . A discrete point is a point in $\mathbf{Z}^{\mathbf{n}}$. A discrete object is a set of discrete points. A Euclidean, also called continuous, object is a set of points in $\mathbf{R}^{\mathbf{n}}$. For a discrete object $A, \bar{A}=\mathbf{Z}^{\mathbf{n}} \backslash A$.

A pixel $V(X), X(x)$ a ID discrete point, is defined by the Euclidean interval $\left[x-\frac{1}{2}, x+\frac{1}{2}\right]$. A pixel $V(X), X(x, y)$ a $2 D$ discrete point, is defined by the Euclidean square $\left[x-\frac{1}{2}, x+\frac{1}{2}\right] \times\left[y-\frac{1}{2}, y+\frac{1}{2}\right]$. A voxel $V(X), X(x, y, z)$ a $3 \mathrm{D}$ discrete point, is defined by the Euclidean cube $\left[x-\frac{1}{2}, x+\frac{1}{2}\right] \times\left[y-\frac{1}{2}, y+\frac{1}{2}\right] \times\left[z-\frac{1}{2}, z+\frac{1}{2}\right]$. Each pixel (voxel) has a unique corresponding discrete point and vice-versa.

The Supercover $S^{\omega}$ of a Euclidean object $S$ is the set of all the discrete points $X$ with corresponding pixel (voxel) $V(X)$ such that $V(X) \cap S \neq \varnothing$.

For a $2 D$ point $A(x, y), A_{x}=(x)$ and $A_{x}=(y)$ are $1 D$ points resulting of the orthogonal projections of $A$ on $O x$ and $O y$. For a $3 \mathrm{D}$ point $A(x, y, z), A_{x}=(x), A_{y}=(y), A_{z}=(z)$ are $1 \mathrm{D}$ points resulting of the orthogonal projections of $A$ on $O x, O y$ and $O z$ respectively. For a 3D point $A(x, y, z), A_{x y}=(x, y), A_{x z}=(x, z)$ and $A_{y z}=(y, z)$ are $2 \mathrm{D}$ points resulting of the orthogonal projections of $A$ on $O x y, O x z$ and $O y z$ respectively.

Let $T$ be a $2 D$ discrete object, then $T^{x}=((x, y, z) \quad(y, z) \in T$ and $x \in Z) ; T=((x, y, z)$ $(x, z) \in T$ and $y \in Z)$ and $T^{2}=\{(x, y, z) \mid(x, y) \in T$ and $z \in Z\}$.

Two discrete points $X\left(x_{1}, \ldots, x_{n}\right)$ and $Y\left(y_{1}, \ldots, y_{n}\right)$ are two $k$-neighbours if $\left|x_{i}-y_{i}\right| \leq I$ for $i \in[1, n]$ and $k \leq n-\sum_{i=1}^{n}\left|x_{i}-y_{i}\right|$. Two discrete points are $k$-adjacent if they are $k$ neighbours but not equal. The proposed notation is adapted to extensions to higher dimensions. $A k$-path in a discrete object $A$ is a sequence of discrete points all in $A$ such that consecutive pairs of points are $k$-neighbours. A discrete object $A$ is $k$-connected if there is a $k$-path between two arbitrary points in $A$. For $B$ a subset of a discrete object $A$. $B$ is $k$-separating in $A$ if $A \cup B$ is not $k$-connected. A discrete object is said to be $k$ separating if it is $k$-separating in $Z^{n}$. Let $A$ be a $k$-separating discrete object such that $\bar{A}$ has exactly two $k$-connected components. $A$-simple point in $A$ is a discrete point $p$ such that $A \varphi$ is $k$-separating. A simple point in $A$ is a discrete point that is a $k$-simple point for some $k$.

Let's consider three different Euclidean points $A, B$, and $C$. The Euclidean line containing $A$ and $B$, is noted $A B$. The Euclidean line segment joining $A$ to $B$ is noted $(A B)$. The Euclidean $3 D$ plane containing $A, B$, and $C$ is noted $A B C$. The Euclidean triangle with vertices $A, B$ and $C$ is noted $(A B C)$. 
A discrete straight line and a discrete plane have been analytically defined by J.-P. Reveillès in the discrete domain as solutions of a double Diophantine inequation [Reve9 1]:

A discrete 2D line is defined by: $L(d, a, b, \omega)=\left\{(x, y) \in Z^{2} \mid 0 \leq a x+b y+d<\omega\right\}$;

A discrete 3D plane is defined by: $P(d, a, b, c . \omega)=\left\{(x, y, z) \in \mathbf{Z}^{3} \mid 0 \leq a x+b y+c z+d<\omega\right\}$

where $a, b, c_{r} d \in \mathbf{Z}^{\mathrm{n}}, \omega \in \mathbf{N}^{*}, \operatorname{gcd}(a, b)=1$ for the $2 \mathrm{D}$ line and $\operatorname{gcd}(a, b, c)=1$ for the 3D plane.

The coefficients of the discrete analytical line (resp. plane) (and also the components of the normal vector) are $a, b$ (and $c$ ). $\omega$ is called the arithmetical thickness and $d$ the translation constant of the line (plane). $\Pi(L, X)=a x+b y+d$ and $\Pi(P, X)=a x+b y+c z+d$ are called the control value of $L$ and $P$ respectively in $X$.

We can suppose without loss of generality that $\operatorname{gcd}(a, b)=l$ for a $2 \mathrm{D}$ discrete line because if $g c d(a, b)=u>l$ then $L(d, a, b, \omega)=L\left(\left\lfloor\frac{d}{u}\right\rfloor, \frac{u}{u}, \frac{b}{u}, \omega^{\prime}\right)$ where $\omega^{\prime}=\left\lfloor\frac{\omega}{u}\right\rfloor+1$ if $\left\{\frac{\omega}{u}\right\}>\left\{\frac{l}{u}\right\}$ and $\omega^{\prime}=\left\lfloor\frac{\omega}{t t}\right\rfloor$ else. An equivalent result is verified for the discrete $3 \mathrm{D}$ plane.

A discrete object is said to be $k$-tinnel free if a $k$-path can "go through" the discrete object without intersection. In our case, a discrete analytical 2D line $T=L(d, a, b, \omega)$ (resp. 3D plane $T=P(d, a, b, c, \omega))$ has a $\mathrm{k}$-tunnel if there exists two $\mathrm{k}$-neighbour discrete points $A$ and $B$ satisfying $\Pi(T, A)<0$ and $\Pi(T, B) \geq \omega$. A discrete object that is 0 -tunnel free is said to be tunnel free.

A 2D discrete line $L(d, a, b, \omega)$ is tunnel free if and only if $\omega \geq|a|+|b|$; it is tunnel free and without simple points if and only if $\omega=|a|+|b|$ [Reve9 I]. A 3D discrete plane $P(d, a, b, c, \omega)$ is tunnel free if and only if $\omega \geq|a|+|b|+|c|$; it is tunnel free and without simple points if and only if $\omega=|a|+|b|+|c|$ [Andr92] [A.S95].

$B o x 1 d(A, B)$, where $A\left(x_{A}\right)$ and $B\left(x_{B}\right)$ are ID Euclidean points, is the Euclidean interval $\left[\min \left(x_{A}, x_{B}\right), \max \left(x_{A}, x_{B}\right)\right]$. $B o x 2 d(A, B)$, where $A\left(x_{A}, y_{A}\right)$ and $B\left(x_{B}, y_{B}\right)$ are 2D Euclidean points, is the Euclidean rectangle $B$ ox $2 d(A, B)=B$ oxld $\left(A_{x}, B_{r}\right) \times B$ oxld $\left(A_{1}, B_{1}\right)$. $B o x 3 d(A, B)$, where $A\left(x_{A}, y_{A}, z_{A}\right)$ and $B\left(x_{B}, y_{B}, z_{B}\right)$ are $3 D$ Euclidean points, is the Euclidean box defined by $\operatorname{Box} 3 d(A, B)=\operatorname{Box} I d\left(A_{x^{\prime}} B_{x}\right) \times \operatorname{Box} I d\left(A_{1}, B_{v}\right) \times \operatorname{Box} I d\left(A_{z^{\prime}} B_{z}\right)$.

A 2D discrete point (3D discrete point) in a 1-path (resp. 2-path) $L$ is called a Jordan point if and only if it has exactly two l-adjacent (resp. 2-adjacent) points in $L$. A 1-path (resp. 2-path) $L$ in $2 D$ (resp. 3D) is called a Jordan curve (also called by some authors a simple curve) if and only if each point of $L$ is a Jordan point. An umbrella at a point $Y$ in a 2D 1 . path (resp. 3D 2-path) is a triple $(X, Y, Z)$, where $X$ and $Z$ are the two 1-adjacent (resp. 2adjacent) points to $Y$ in the path.

An umbrella at a point $Y$ in a 3D 2-connected plane is a circular permutation (up to its orientation) of unit squares incident to $Y$, included in the plane, and such that (1) two consecutive squares in the permutation share a common edge, and (2) only two squares share a common edge. A point in a 2-connected discrete plane $P$ is called a Jordan point if and only if it has exactly one umbrella incident to it. If any point of a 2-connected plane is a Jordan point then the plane is a 2-manifold [Fra95b] [Fra95a]. 


\section{Supercover Primitives: Properties and Algorithms}

In this section, we are going to examine the properties of several ID, 2D, and 3D Supercover primitives, and provide algorithms for them. The main mathematical results are the theorems. A Supercover primitive can have two main types of algorithms. The first type is a generation algorithm that generates all the discrete points of the Supercover of a Euclidean primitive. The second type is a localization algorithm that tells us if a given discrete point belongs or not to the Supercover of a Euclidean primitive. While all the primitives have an associated localization algorithm, only the finite sized primitives have an associated generation algorithm. An example for this is the $2 \mathrm{D}$ line. We propose a generation and localization algorithm for the Supercover of a $2 \mathrm{D}$ line segment joining two points but only a localization algorithm for the Supercover of a complete $2 \mathrm{D}$ line. Note that by brute-force, it is always possible to generate the Supercover of a primitive by localizing all its points in a given finite sized region.

This section is divided in three subsections corresponding to the Supercovers of boxes and points, and for 2D and 3D primitives. We don't give all the details of the algorithms since it would be too long but the complete algorithms are available, in a C-language source file, at the Supercover Home Page:

http: //dpt-info.u-strasbg. Er/-nehlig/Supercover.htmI

The aim of this feature is to allow easy access to a basic Discrete Geometry Library for comparisons, tests and developments. Future revisions will include primitives for more complex objects, extensions on higher dimensions as well as bubble-free Supercovers.

\section{I Supercover Boxes and Points}

Let's consider for what follows the $3 D$ rational points $A\left(x_{A} / R, y_{A} / R_{z} z_{A} / R\right)$,

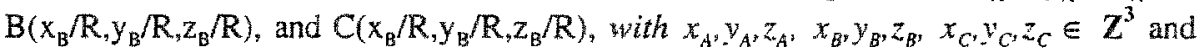
$R \in \mathrm{N}^{*}$. Let's consider also the 3D discrete point $Q\left(x_{Q^{\prime}} y_{Q^{\prime}} z_{Q}\right), x_{Q^{\prime}} y_{Q^{\prime}} z_{Q} \in \mathbf{Z}$. This point is used in the following localization algorithms.

Proposition 1: Supercover Boxes:

ID box: $\left(A_{x} B_{x^{\prime}}\right)^{\omega}=\operatorname{Boxld}\left(A_{x^{\prime}} B_{x}\right)^{\omega}=\left[\left[\frac{2 \min \left(x_{A}, x_{B}\right)+R-1}{2 R}\right),\left[\frac{2 \max \left(x_{A}, x_{B}\right)+R}{2 R}\right]\right.$.

2D box: Box $2 d\left(A_{\mathrm{r}}, B_{\mathrm{rv}}\right)^{\omega}=\operatorname{Box} l d\left(A_{x}, B_{x}\right)^{\omega} \times B o x l d\left(A_{1}, B_{1}\right)^{\omega}$.

3D box: $\operatorname{Box} 3 d(A, B)^{\omega}=\operatorname{Box} 2 d\left(A_{x,} B_{\mathrm{r}}\right)^{\omega} \times \operatorname{Box} / d\left(A_{z}, B_{z}\right)^{\omega}$.

$2 \mathrm{D}$ triangle bounding box:

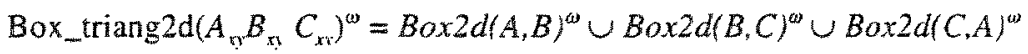

$$
\begin{aligned}
& \text { Box_triang2d }\left(A_{x y} B_{\mathrm{xr}} C_{\mathrm{xx}}\right)^{\omega x}=\operatorname{Box} I d\left(\min \left(A_{\mathrm{x}^{\prime}} B_{x^{\prime}} C_{x}\right), \max \left(A_{x^{\prime}} A_{y}, A_{y}\right)\right)^{\omega} \\
& \times \operatorname{Box}) d\left(\min \left(A_{,}, B_{1,} C_{v}\right), \max \left(A_{y^{\prime}} B_{r^{\prime}} C_{y}\right)\right)^{o} \text {. }
\end{aligned}
$$

3D triangle bounding box:

$$
\begin{aligned}
& \text { Box_triang } 3 \mathrm{~d}(A B C)^{\mathrm{an}}=B o \times 3^{3} d(A, B)^{\omega} \cup B o \times 3 d(B, C)^{\omega} \cup B o x 3 d(C, A)^{\omega} \\
& \text { Box_triang } 3 \mathrm{~d}(A B C)^{\omega}=B o x_{-} \text {triang } 2 d\left(A_{11}, B_{11}, C_{\mathrm{n}}\right)^{\omega}
\end{aligned}
$$

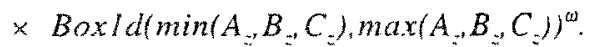


The Supercover of a discrete point $s$ simply a particular case of an interval, therefore:

Proposition 2: Supercover of a ID point $A: A^{(0)}=B o x l d(A, A)^{\omega}$.

Supercover of a 20 point: $A^{\omega}=\operatorname{Box} 2 d(A, A)^{\omega}$.

Supercover of a $3 D$ point: $A^{\omega}=\operatorname{Box} 3 d(A, A)^{\omega}$.

The Supercover of a ID (resp. 2D, 3D) point is composed of 1 or 2 (resp. 1 or 2 or 4.1 or 2 or 4 or 8 ) discrete points. A localization algorithm and a generation algorithm for points are immediately deduced.

\section{2 Dimension 2}

In dimension 2, we study the supercovers of a $2 \mathrm{D}$ line defined by two rational points, a $2 \mathrm{D}$ line segment joining two rational points and a $2 \mathrm{D}$ triangle defined by three rational points. Let's consider for what follows the $2 \mathrm{D}$ rational points $A\left(x_{A} / R, y_{A} / R\right), B\left(x_{B} / R\right.$, $\left.y_{B} / R\right)$, and $C\left(x_{C} / R, y_{C} / R\right)$, with $x_{A}, y_{A}, x_{B}, y_{B}, x_{C}, y_{C} \in \mathbf{Z}$ and $R \in \mathbf{N}^{*}$. Let's consider also the 2D discrete point $Q\left(x_{Q}, y_{Q}\right)$, with $x_{Q}, y_{Q} \in \mathrm{Z}$. This point is used in the following localization algorithms.

\subsubsection{D Supercover line}

Let's consider the two points $A$ and $B$ and define $d x=x_{B}-x_{A}$ and $d y=y_{B}-y_{A}$.

If $d x=d y=0$ it means that $A=B$ and therefore $A B^{\omega}=A^{\omega}$. We'll assume for what follows that $A$ and $B$ are two different points.

Let's define: $a=\frac{d y}{G C D(d x, d y)}$ and $b=\frac{-d x}{G C D(d x, d y)}$ and $d=-a x_{A}-b y_{A}$.

The points $A$ and $B$ belong to the Euclidean line $L: a x+b y+d / R=0$. For what follows we call $\omega_{1}=-\left\lfloor\frac{R(|a|+|b|)+2 d}{2 R}\right\rfloor$ and $\omega_{2}=\left\lfloor\frac{R(|a|+|b|)-2 d}{2 R}\right\rfloor$. The Supercover of $L=A B$ is given by:

Theorem 3: Supercover of a $2 D$ straight line:

$$
A B^{\omega}=\left\{(x, y) \in \mathbf{Z}^{2} \mid \omega_{1} \leq a x+b y \leq \omega_{2}\right\}=L\left(-\omega_{1}, a, b, \omega_{2}-\omega_{1}+1\right) . .
$$

The Supercover $A B^{\omega}$ is a Reveillès $2 D$ discrete line of arithmetical thickness $\omega=\omega_{2}+1$ $\omega$. When $d / R=k$, an integer, and $|a|+|b|$ even or $d / R=k+1 / 2$ and $|a|+|b|$ odd then $\omega$ $=|a|+|b|+1$. The discrete line is then 1 -connected with simple points located at 4 -bubbles. In all the other cases, $\omega=|a|+|b|$ and the line is a 1 -connected Jordan line, that is without simple points; these lines are called standard lines. Note that, if $R=1$, that is, if $A$ and $B$ are integer points, then $\omega_{i}=-\omega_{2}$ : moreover. the control values in $A$ and $B$ are 0 .

A 4-bubble in a 2D (resp. 3D) discrete line is a set of four different integer points $P_{1}, P_{2}, P_{3}, P_{4}$ where $P_{1}$ is a 1 -adjacent (resp. 2-adjacent) of $P_{2}, P_{2}$ of $P_{3}, P_{3}$ of $P_{4}$ and $P_{4}$ of $P$.

Ex. In the Supercover of the Euclidean 2D line $x+y=0$ any point belongs to a 4-bubble.

The same holds for the line $y=1 / 2$, and for the line $x+2 y+1 / 2=0$. 


\section{Proposition 4: Location of t-bubbles:}

Let's suppose that the equation of the Supercover $A B^{\omega}$ is: $\omega_{l} \leq a x+b y \leq \omega_{2}$, with $0 \leq a \leq b$. The Supercover $A B^{\omega}$ has 4-bubbles if and only if $\omega=\omega_{2}+l-\omega_{1}=a+b+l$. The control values at the points of a 4 -bubble are $\omega_{l}, \omega_{2}, \omega_{2}-a$ or $\omega_{1}+a$. The simple points are the points of control value $\omega_{1}$ and $\omega_{2}$.

Sketch of proof: According to [Reve91]. a discrete line is 4-connected, without simple points, if and only if its arithmetical thickness is $a+b$; a Supercover line has thus a bubble if and only if its arithmetical thickness is $a+b+1$. Let $r$ be the remainder at a point with minimum $x$ coordinate and maximum $y$ coordinate, belonging to a given bubble. The remainders at the other three points of the bubble are then $r+a . r+b$, and $r+a+b$, and these 4 numbers have to belong to the $\left[\omega_{1}, \omega_{2}\right]$ interval. The only solution is $r=\omega_{i}$, and $r+a+b=\omega_{2}$. The last part of the proposition can similarly be obtained.

Proposition 5: Let $L$ be a Supercover 2D line, and let $p$ a point of $L$. If $p$ does not belong to a 4-bubble, then $p$ is a Jordan point.

A line is not of finite size, therefore we'll only propose a localization algorithm for this primitive. A localization algorithm Super_local_line2d(A.B,Q) can be deduced immediately from theorem 3 .

\subsubsection{D Supercover line segment}

It is easy to see that the Supercover of a Euclidean $2 \mathrm{D}$ line segment joining two rational points $A$ and $B$ is given by:

Proposition 6: Supercover of a $2 D$ discrete line segment:

$$
(A B)^{\omega}=A B^{\omega} \cap \operatorname{Box} 2 d(A, B)^{\omega} \text {. }
$$

A localization algorithm Super_local_lineseg $2 \mathrm{~d}(A, B, Q)$ can be immediately deduced.

The design of an efficient generation algorithm Super_gen_lineseg $2 \mathrm{~d}(A, B)$ is however more complicated. Let's consider $d x=x_{B}-x_{A}, d y=y_{B}-y_{A}$, used in the computation of the coefficients of the discrete line $A B^{\omega}$. If $d x$ or $d y$ are null then it means that $A=B$ or that we have a horizontal or a vertical line. All three cases can be handled by Super_gen_box $2 \mathrm{~d}(A, B)$.

Now, let's suppose that $0 \leq d y \leq d x$. This can be obtained by symmetry. The first thing we do is to compute the coefficients $a, b, \omega_{1}, \omega_{2}$ of the $2 \mathrm{D}$ Supercover line according to theorem 3 and the bounding box $B o x 2 d(A, B)^{\omega}=\left[x_{d}, x_{f}\right] \times\left[y_{d}, y_{f}\right]$. We can now use an incremental generation algorithm where $\left(x_{d d^{\prime}} y_{d}\right)$ will be the first point to be generated and $\left(x_{f}, y_{f}\right)$ the last. The control value $\Pi$ of the incremental algorithm is initialized with $\Pi=a x_{d}+b y_{d}$. At each loop of the algorithm we advance $x$ and adjust $P$ by an increment of $a$. As long as we have $P \leq \omega_{2}$ we continue. Once this is not true anymore, we have to increment $y$. In order to detect if there is a 4-bubble, we decrement then $x$ by 2 . If this point belongs to the line then there was a 2-bubble at that point and we generate it. else we simply continue by incrementing $x$ in the regular loop. The algorithms stops when it reaches the point $\left(x_{p}, y\right)$.

\subsubsection{Supercover $2 \mathrm{D}$ triangle}

Let's call $T$ the triangle $T=(A B C)$. Let's first assume that the three points are non aligned because else we have simply $T^{\nu}=(A B)^{\omega} \cup(B C)^{\omega} \cup(C A)^{\omega}$. 
Let's assume the Cartesian equation of the Euclidean line $A B$ is $a, x+b_{1} y+d_{1} / R=0$ defined so that $a_{r} x_{C}+b_{l} y_{C}+d_{l} / R>0$. In the same way, the equation of $B C$ is $a_{2} x+b_{3} y+d \sqrt{ } R=0$ defined so that $a_{2} x_{A}+b_{2} v_{A}+d_{z} / R>0$ and the equation of $C A$ is $a_{3} x+b_{3} y+d_{3} / R=0$ defined so that $a_{3} x_{B}+b_{3} y_{B}+d, R>0$.

Let's consider now the Supercovers of these three lines:

$A B^{\omega}: \omega_{1} \leq a_{1} x+b_{1} y \leq \rho_{1} ; B C^{\omega}: \omega_{2} \leq_{2} x+b_{2} y \leq \rho_{2}$ and $C A^{\omega}: \omega_{3} \leq a_{3} x+b_{3} y \leq \rho_{3}$.

Let's call $H_{k}$ the discrete half space $a_{k} x+b_{k} v \geq \omega_{k}$ and $I_{k}$ the discrete half space $a_{k} x+b_{k} y>\rho_{k}$, $k=1,2,3$, then:

Theorem 7: Supercover of a $2 D$ rational triangle:

$T^{\omega}=(A B C)^{\omega}=H_{1} \cap H_{2} \cap H_{i} \cap$ Box_triang2d $\left(A B C^{\omega}\right)$

$T^{\omega}=(A B C)^{\omega}=\left((A B)^{\omega} \cup(B C)^{\omega} \cup(C A)^{\omega} \cup\left(I_{1} \cap I_{2} \cap I_{3}\right)\right) \cap$ Box_triang2d $(A B C)^{\omega}$.

We call interior of $T^{w}$ the integer set $I_{1} \cap I_{2} \cap I_{3}$; it can be empty. We call boundary of $T^{w}$ the set $\left((A B)^{\omega} \cup(B C)^{\omega} \cup(C A)^{\omega}\right) \cap B o x$ triang2 $\mathrm{d}(A B C)^{\omega}$. Thus, $T^{\omega}$ is the union of its interior and its boundary. Moreover, the interior and the boundary of $T^{\omega}$ do not intersect.

Note that the intersection with the Supercover of the bounding box Box_triang $2 \mathrm{~d}(A B C)^{10}$ is necessary to define the boundary of $T^{\omega}$, because the intersection of $(A B)^{\omega}$ and $(B C)^{\omega}$, for example, can contain points outside $T^{\text {wo }}$, as shown on the following example: $A B$ is the line $y=0, A C$ is $x-9 y=0$, and $A=(0,0), B$ and $C$ have positive abscissa; the point $(-1$, 0 ) belongs to $A B^{\omega} \cap A C^{\omega}$ but not to $T^{\omega}$.

A localization algorithm Super_local_triang $2 \mathrm{~d}(A, B, C, Q)$ is simple to design with the results of proposition 6 and theorem 7 .

The design of a generation algorithm is slightly more complicated. We can of course generate the triangle by brute-force by testing what points of the Supercover of the bounding box belong to the Supercover triangle with help of the localization algorithm. This is however not efficient. A more efficient method consists in an adaptation of the classical scanline filling algorithm. We won't describe here the algorithm which would be too long. Let's just remark that if we have a discrete line $L: \omega_{1} \leq a_{1} x+b_{1} y \leq \rho_{1}$, with $a>0$, then for a given $y=y_{i}$ we have $-\left\lfloor\frac{b y_{i}-\omega_{1}}{a}\right\rfloor \leq x \leq\left\lfloor\frac{\omega_{2}-b y_{i}}{a}\right\rfloor$. By doing that for two lines it is easy to determine a span of pixels to fill up.

\subsection{Dimension 3}

In dimension 3, we study the supercovers of a 2D line defined by two 2D rational points. a 3D line segment joining two rational 3D points, a 3D plane defined by three 3D rational points and a $3 \mathrm{D}$ triangle defined by three rational $3 \mathrm{D}$ points. Let's consider for what follows the $3 D$ rational points $A\left(x_{A} / R, y_{A} / R, z_{A} / R\right), B\left(x_{B} / R, y_{B} / R, z_{B} / R\right)$, and $\mathrm{C}\left(\mathrm{x}_{\mathrm{B}} / \mathrm{R}, \mathrm{y}_{\mathrm{B}} / \mathrm{R}, \mathrm{z}_{\mathrm{B}} / \mathrm{R}\right)$, with $x_{A}, y_{A}, z_{A}, x_{B}, y_{B}, \tilde{z}_{B}, x_{C}, y_{C}, z_{C} \in \mathbf{Z}^{3}$ and $R \in \mathrm{N}^{*}$. Let's consider also the $3 \mathrm{D}$ discrete point $Q\left(x_{Q}, y_{Q}, z_{Q}\right), x_{Q}, y_{Q}, z_{Q} \in \mathrm{Z}$. This point is used in the following localization algorithms.

\subsubsection{Supercover of a 3D straight line}

Let's consider the two points $A$ and $B$ and define $d x=x_{B}-x_{d}, d y=y_{B}-y_{4}$ and $d z=z_{B}-x_{-1}$. 
If $d x=d y=d z=0$ it means that $A=B$ and therefore $A B^{\omega}=A^{w}$. We'll assume for what follows that $A$ and $B$ are two different points.

Theorem 8: Supercover of a $3 D$ straight line:

$$
\begin{aligned}
& A B^{\omega}=\left(A_{x} B_{y}{ }^{\omega}\right) \cap\left(A_{v} B_{r:}{ }^{\omega}\right)^{\prime} \cap\left(A_{1} B_{v:}{ }^{\omega}\right)^{r} \\
& A B^{\omega}=P\left(-\omega_{1}, a_{1}, b_{1}, 0, \rho_{1}-\omega_{1}+1\right) \cap P\left(-\omega_{2}, a_{2}, 0, b_{2}, \rho_{2}-\omega_{2}+1\right) \cap P\left(-\omega_{3}, a_{1} a_{3}, b_{3}, \rho_{3}-\omega_{3}+1\right) \\
& \text { where } A_{x r} B_{\mathrm{n}}{ }^{\omega}=L\left(-\omega_{1}, a_{1}, b_{l}, \rho_{1}-\omega_{i}+1\right): A_{x_{2}} B_{1:}{ }^{\omega}=L\left(-\omega_{2}, a_{2}, b_{2}, \rho_{2}-\omega_{2}+1\right) \\
& \text { and } A_{1 z} B_{y z}{ }^{\omega}=L\left(-\omega_{3}, a_{3}, b_{3}, p_{3}, \omega_{3}+1\right) \text {. }
\end{aligned}
$$

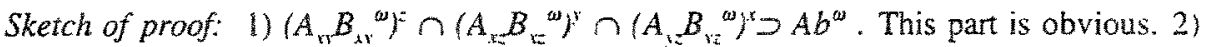
$A b^{\omega} \supset\left(A_{x y} B_{x y}{ }^{\omega}\right)^{*} \cap\left(A_{x z} B_{x z}{ }^{\omega}\right)^{\prime \prime} \cap\left(A_{y} B_{y z}{ }^{\omega}\right)^{x}$. This inclusion is due to following lemma: Let $D$ be a Euclidean $3 D$ line and let $(u, v, w)$ be an integer coordinate point such as the projection of $D$ on the $(x, y)$ (resp. $(y, z),(z, x)$ ) plane intersects the pixel of centre $(u, v)$ (resp. $(v, w),(w, u))$ of this plane, then $\mathrm{D}$ intersects the voxel of centre $(u, v, w)$.

The analytical definition of the Supercover 3D lines is based on three $2 \mathrm{D}$ lines (respectively in the planes $x y, y z$ and $z x$ ) or three planes. It can be proved that two of them are, generally, not enough, as it can be seen in Figure 1 . This is a major difference with classical definitions. The fact that the intersection of three discrete planes defines a discrete line creates a symmetry in the 3D line that does not exist in classical definitions.

It is easy to see that a 3D Supercover line is 2-connected; it can be a 2-connected Jordan curve (that is without a simple point); but it can have simple points if it contains 4 bubbles, like a 2D Supercover line, or 8-bubbles. An 8-bubble in a 3D line or plane is a set of eight discrete points forming a $1 \times 1 \times 1$ unit cube. A 8-bubble in a 3D segment is visible on the Figure 2. In the same Figure and in Figure 1, a 4-bubble in a 3D segment is also visible.

A line is not of finite size, therefore we'll only propose a localization algorithm for this primitive. A localization algorithm Super_local_line $2 \mathrm{~d}(A, B, Q)$ can be deduced immediately from theorem 8 .

\subsubsection{Supercover of a 3D straight line segment}

It is easy to see that the Supercover of a Euclidean 3D line segment joining two rational points $A$ and $B$ is given by

Proposition 9: Supercover of a 3D line segment:

$$
(A B)^{\omega}=A B^{\omega} \cap B \circ x 3 d(A, B)^{\omega} \text {. . }
$$

Figure 1 shows an example of a Supercover 3D segment.

Proposition 10: Supercover of a $2 D$ line segment in $3 D$ space:

Let's suppose that $z_{B}{ }^{-z_{d}}=0$; then $(A B)^{\omega}=\left(A_{n} B_{n}\right)^{\omega} \times B$ Baxld $\left(A_{3}, B_{z}\right)^{\omega}$. .

A localization algorithm Super_local_lineseg $2 \mathrm{~d}(A, B, Q)$ can be immediately deduced. The design of an efficient generation algorithm Super_gen_lineseg $3 \mathrm{~d}(A, B)$ is, however. more complicated. Let's consider $d x=x_{B}-x_{A} d y=y_{B}-v_{A}$, and $d z=z_{B}-z_{A}$. If two or three of the three $d x, d y$ and $d z$ are null then it means that $A=B$ or that we have a line parallel to an axis. All these cases can be handled by Super_gen_box3d( $A, B)$. Let's suppose that only one is equal to zero, $d z=0$ for instance. We generate a $2 D$ segment and apply proposition 6. 
Now, let's suppose that $0 \leq d z \leq d y \leq d x$. We know from theorem 8 that a point $(x, y, z)$ belongs to the 3D line if $(x, y)$ belongs to $A_{1} B_{\mathrm{x} y}{ }^{\omega}$, if $(y, z)$ belongs to $A_{y z} B_{v:}{ }^{\omega}$ and if $(x, z)$ belongs to $A_{x z} B_{x z}{ }^{\omega}$. What we do therefore is to run three $2 \mathrm{D}$ line segment generation algorithms together. At each step we verify that all three conditions are verified.

\subsubsection{Supercover 3D plane}

Let's consider the three rational $3 \mathrm{D}$ points $A, B$, and $C$.

Let's define $d x=\left(y_{B}-y_{A}\right)\left(z_{C}-z_{A}\right)-\left(y_{C}-y_{A}\right)\left(z_{B}-z_{A}\right), \quad d y=\left(z_{B}-z_{A}\right)\left(x_{C}-x_{A}\right)-\left(z_{C}-z_{A}\right)\left(x_{B}-x_{A}\right), \quad$ and $d z=\left(x_{B}-x_{A}\right)\left(y_{C}-y_{A}\right)-\left(x_{C}-x_{A}\right)\left(y_{C}-y_{A}\right)$.

If $d x=d y=d z=0$ then it means that $A B$ and $A C$ are collinear and do not define a $3 D$ plane but a $3 \mathrm{D}$ line or a $3 \mathrm{D}$ point. We'll assume for what follows that $A, B$ and $C$ are three non aligned points.

Let's define: $a=\frac{d x}{G C D(d x, d y, d z)}, b=\frac{d y}{G C D(d x, d y, d z)}, c=\frac{d z}{G C D(d x, d y, d z)}$ and $d=-a x_{A}-b y_{A}$ $c z_{A}$. The points $A, B$ and $C$ belong to the Euclidean plane $P: a x+b y+c z+d / R=0$.

We note $\omega_{1}=-\left\lfloor\frac{R(|a|+|b|+|c|)+2 d}{2 R}\right\rfloor$ and $\omega_{2}=\left\lfloor\frac{R(|a|+|b|+|c|)-2 d}{2 R}\right\rfloor$.

The Supercover of $P=A B C$ is then given by:

Theorem 11: Supercover of a $3 D$ plane:

$P^{\omega}=A B C^{\omega}=\left\{(x, y, z) \in \mathbf{Z}^{3} \mid \omega_{1} \leq a x+b y+c z \leq \omega_{2}\right\}=P\left(-\omega_{i}, a, b, c, \omega_{2}-\omega_{1}+1\right) . \bullet$

The Supercover $P^{\omega}$ is a Reveillès 3D discrete plane of arithmetical thickness $\omega=\omega_{2}+l-\omega_{l}$. When $\mathrm{d} / \mathrm{R}=k, k$ integer, and $|a|+|b|+|c|$ even or $d / R=k+1 / 2, k$ integer, and $|a|+|b|+|c|$ odd then $\omega=|a|+|b|+|c|+l$. The discrete plane is 2 -connected, tunnel free and it has 8 -bubbles. In all the other cases, $\omega=|a|+|b|+|c|$. The discrete plane is then 2-connected, tunnel free. and without simple points (it is a 2-manifold [Fra95b] or [Fra95a]). Note that, if $R=1$, that is, if $A, B$ and $C$ are integer points, then $\omega_{1}=-\omega_{2}$; moreover, the control values in $A$, $B$ and $C$ are 0 .

\section{Proposition 12: Control value at a 8-bubble:}

Let's suppose that the equation of the Supercover $P^{\omega_{1}}$ : $\omega_{1} \leq a x+b y+c z \leq \omega_{2}$, with $0 \leq a \leq b \leq c$. The Supercover $P^{\omega}$ contains 8-bubbles if and only if $\omega=\omega_{2}+1-\omega_{1}=$ $a+b+c+1$. The control values at the points of a 8-bubble are $\omega_{l}, \omega_{1}+a, \omega_{1}+b, \omega_{1}+a+b$, $\omega_{1}+c, \omega_{1}+c+a, \omega_{1}+c+b, \omega_{1}+a+b+c=\omega_{2}$.

Proposition 13: Let $P$ be a Supercover plane, and let $p$ a point of $P$. If $p$ does not belong to a 8-bubble then $p$ is a Jordan point.

Because the intersection of two non parallel Euclidean planes is a Euclidean line we have:

Theorem 14: Intersection of $3 D$ Supercover planes:

Let's consider two 3D rational points $A$ and $B$. The intersection of the supercovers of all the Euclidean planes containing $\mathrm{AB}$ is equal to $A B^{(1)}$.

Since a $3 \mathrm{D}$ plane is not finite. we propose only a localization algorithm called Super_local_plane $3 \mathrm{~d}(A, B, C, Q)$. With help of theorem 11 the algorithm design is straight forward. 


\subsubsection{Supercover $3 \mathrm{D}$ triangle}

Let's call $T$ the triangle $T=(A B C)$. Let's first assume that the three points are non aligned because else we have simply $T^{\omega}=(A B)^{\omega} \cup(B C)^{\omega} \cup(C A)^{\omega}$.

Theorem 15: Supercover of a 3D triangle:

$$
T^{\omega}=(A B C)^{\omega}=\left(T_{x y}^{\omega}\right) \cap\left(T_{x z}^{\omega}\right)^{\prime} \cap\left(T_{y z}^{\omega}\right)^{\prime} \cap A B C^{w} .
$$

A Supercover $3 \mathrm{D}$ triangle is shown in Figure 2.

Note that, like in theorem 8, two of the three triangles $T_{x y}, T_{x \rightarrow}, T_{x z}$ are not enough, as it can be shown on examples. Furthermore, as any polygon can be tiled using triangles. the Supercover of a polygon is defined as the union of the Supercovers of a tiling set of triangles.

A localization algorithm Super_local_triang $3 \mathrm{~d}(A, B, C, Q)$ is easy to design with theorem 15. The generation algorithm Super_gen_triang $3 \mathrm{~d}(A, B, C)$ of a $3 \mathrm{D}$ triangle is actually much easier to implement than the $2 \mathrm{D}$ algorithm. After having dealt with the degenerate cases and so being sure that the three points are not aligned we can compute the coefficients of the Supercover of the Euclidean plane $P=A B C$.

Let's suppose that the Supercover of $P$ is equal to $P^{\omega}: \omega_{1} \leq a x+b y+c z \leq \omega_{2}$. Let's suppose here, without loss of generality that $c>0$. Now we generate the 2D Supercover triangle $T_{x y}^{\omega}$ and for each $\left(x_{i} y_{k}\right)$ belonging to $T_{x y}^{\omega}$ we compute all the corresponding $\left(x_{i}, y_{p}, z_{k}\right)$ belonging to $p^{\omega}$. We know that $-\left\lfloor\frac{a x_{i}+b y_{i}-\omega_{1}}{c}\right\rfloor \leq z_{k} \leq\left\lfloor\frac{\omega_{2}-a x_{i}-b y_{i}}{c}\right\rfloor$. For each $\left(x_{i}, y_{i}, z_{k}\right)$ we do a

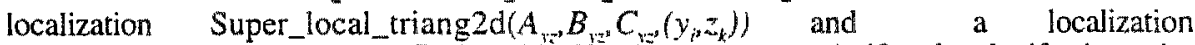

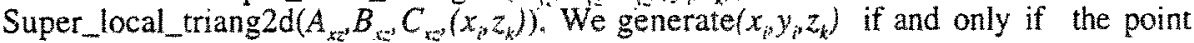
$\left(y_{i}, z_{k}\right)$ belongs to $T_{y z}^{\omega}$ and if $\left(x_{i}, z_{k}\right)$ belongs to $T_{x=}^{\omega}$.

\section{Conclusion}

We have defined the Supercover of continuous objects. We have then found out analytical description, by the way of inequalities (sets of Diophantine equations), of the basic primitives of the geometry: $2 \mathrm{D}$ straight lines, segments and triangles, 3D straight lines, planes and triangles. We have given geometric properties concerning the arithmetical thickness of the lines and of the planes, and the bubbles. Basic algorithms for localization and for generation have been given; they are adaptations of classical algorithms for continuous primitives.

With these primitives and algorithms a discrete geometric modelling whose primitives are polygons and polyhedra is possible; the first results are shown in [ANF97].

New discrete geometric problems are in order. The main one is: is it possible to use discrete $2 \mathrm{D}$ straight lines, and discrete planes, whose thickness is lesser than that of Supercovers, and verifying analogous theorems, especially an analogue of theorem 14 ? Examples show that it is not the case of naive planes of [Reve91] and naive lines of [FR95]. 


\section{References}

[Andr92] E. Andres, Cercles discrets et Rotations discretes [Discrete Cercles and Discrete Rotations], Ph.D. Thesis, Université Louis Pasteur, Strasbourg (France). Dec. 1992 (in French).

[AS95] E. Andres, and C. Sibata, Discrete hyperplanes in arbitrany dimensions. to be published in CVGIP-GMIP.

[ASA96] E. Andres, C. Sibata, and R. Acharya, Supercover 3D Polygon. Proc. Int. Workshop on Discrete Geometry for Computer Imagery, LNCS, vol.1176, pp. 237-242.

[ANF97] E. Andres. Ph. Nehlig, and J. Françon, Tunnel-free Supercover 3D polygons and polyhedra, Eurographics'97, BUDAPEST, Hungary, Sept 4-8 (1997). Computer Graphics Forum (Blackwell Publishers), vol 16(3), Conference issue, pp. C3-C13.

[Cohe95] D. Cohen and A. Kaufman. Fundamentals of surface voxelization. CVGIPGMIP, 57(6), Nov.95, pp.453-461.

[Fra95a] J. Françon, Arithmetic planes and combinatorial manifolds, $5^{\text {th }}$ International Conference Discrete Geometry in Computer Imagery, Clermont-Ferrand, 25-27 Sept. 1995, pp. 209-217.

[Fra95b] J. Françon, Sur la topologie d'un plan arithmétique [About the topology of the arithmetical plane], Theor. Comp. Sc. 156, 1996, pp. 159-176 (in French).

[FR95] O. Figueiredo, and 3.-P. Reveillès, $A$ contribution to $3 D$ digital lines, $5^{\text {th }}$ International Conference Discrete Geometry in Computer Imagery, Clermont-Ferrand. 25-27 Sept. 1995, pp. 187-198.

[Reve91] J.-P. Reveillès, Géométrie Discrète, calcul en nombres entiers et algorithmique [Discrete Geometry, Integer Number Calculus, and Algorithmics], Thèse d'état. Université Louis Pasteur, Strasbourg (France), Dec. 1991 (in French). 


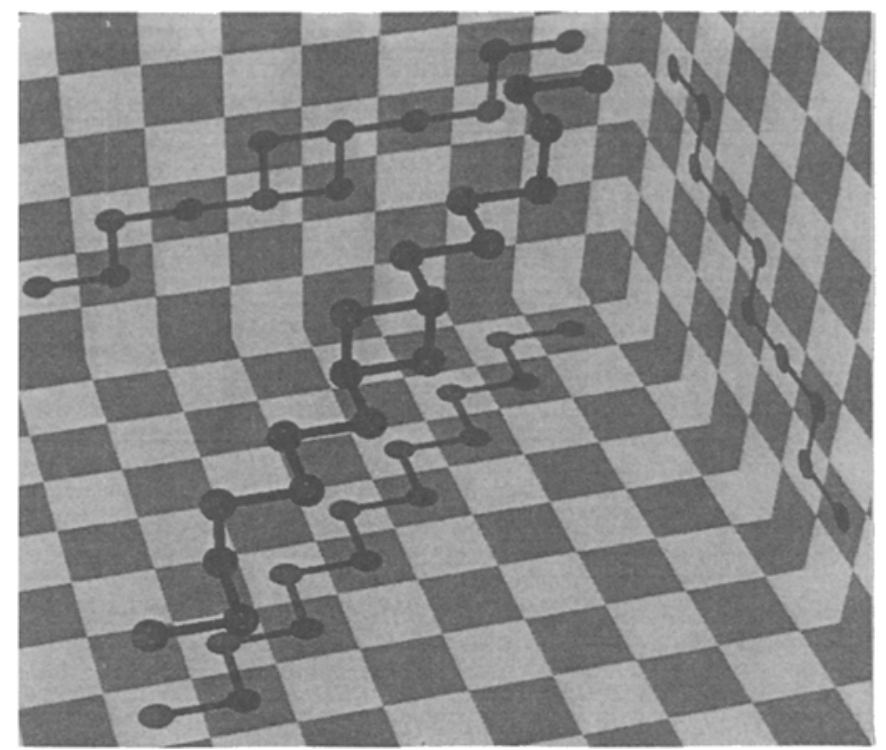

Figure 1: The Supercover of a $3 \mathrm{D}$ segment

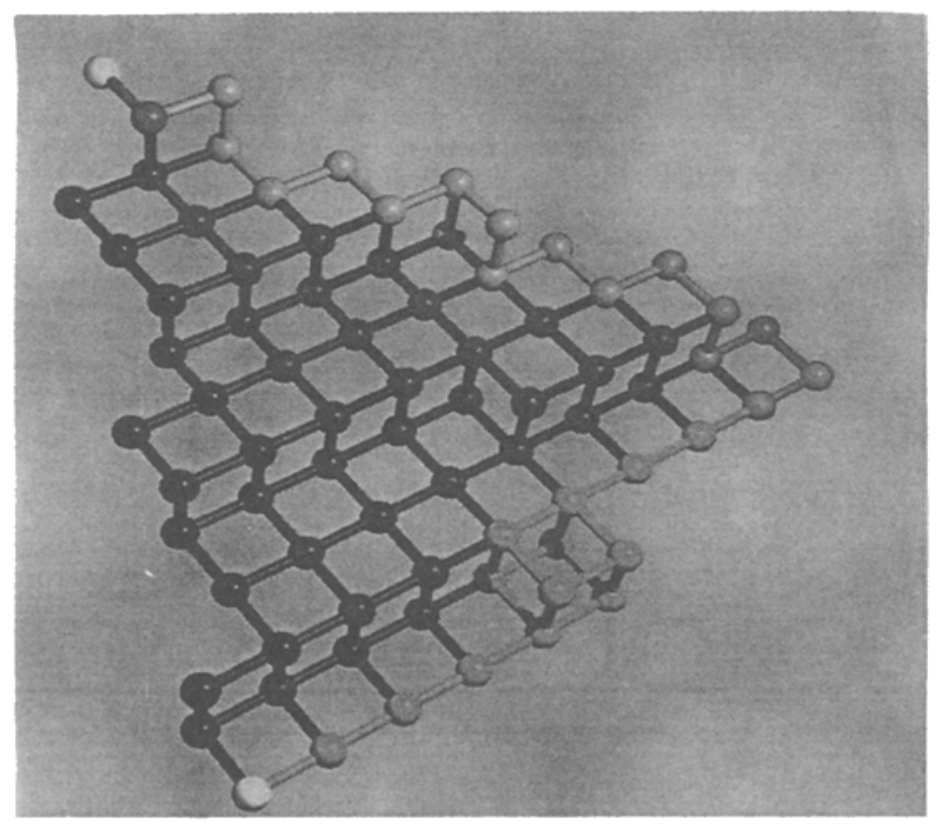

Figure 2: The Supercover of a 3D triangle

The vertices are of one color, the edges are each of a different colot, the interior is of another color 